Cette article analyse l'origine physique et la forme des différentes trajectoires d'un ballon de football.

\section{En l'absence de gravité et} d'air, celle-ci est une droite. Avec gravité, la droite s'incurve vers la parabole galiléenne.

\section{L'aérodynamique enrichit} ces deux comportements classiques et fait apparaître des zigzags, des paraboles tronquées et des spirales.

Nous discutons les propriétés de ces différentes trajectoires et les phases de jeu qui permettent de les observer.

\title{
Le football et ses trajectoires
}

Guillaume Dupeux (guillaume.dupeux@espci.fr), Caroline Cohen, Anne Le Goff, David Quéré et Christophe Clanet (clanet@ladhyx.polytechnique.fr)

LadHyX, UMR CNRS 7646, École polytechnique, 91128 Palaiseau, et PMMH, UMR CNRS 7636, ESPCI, 10 rue Vauquelin, 75005 Paris

Les jeux de balles nous accompagnent depuis au moins 3500 ans [1]. Ils font partie de notre évolution et nous les retrouvons sur tous les continents: Tsu Chu en Chine, Kemari au Japon, Episkiros en Grèce, Pok a tok en Amérique du Sud. Une de leurs versions contemporaines est le football. Il se pratique à deux équipes de onze joueurs, avec un ballon rond de $450 \mathrm{~g}$ et $22 \mathrm{~cm}$ de diamètre (fig. 1a), et sur un terrain d'une centaine de mètres, dont les dimensions précises sont indiquées sur la figure $1 \mathrm{~b}$.

Les études sur la physique du football portent principalement sur la mécanique et la statistique. Les pionniers en statistique sont Reep et Benjamin [2], qui étudient la probabilité de perdre le ballon en fonction du nombre de passes. Cette étude est à l'origine du jeu long pratiqué par certaines équipes, à commencer par la Norvège lorsque Charles Reep deviendra conseiller technique de l'équipe nationale. Côté mécanique, on s'est intéressé essentiellement aux trajectoires et aux impacts [3].

Nous discutons ici les différents types de trajectoires et leur origine physique.

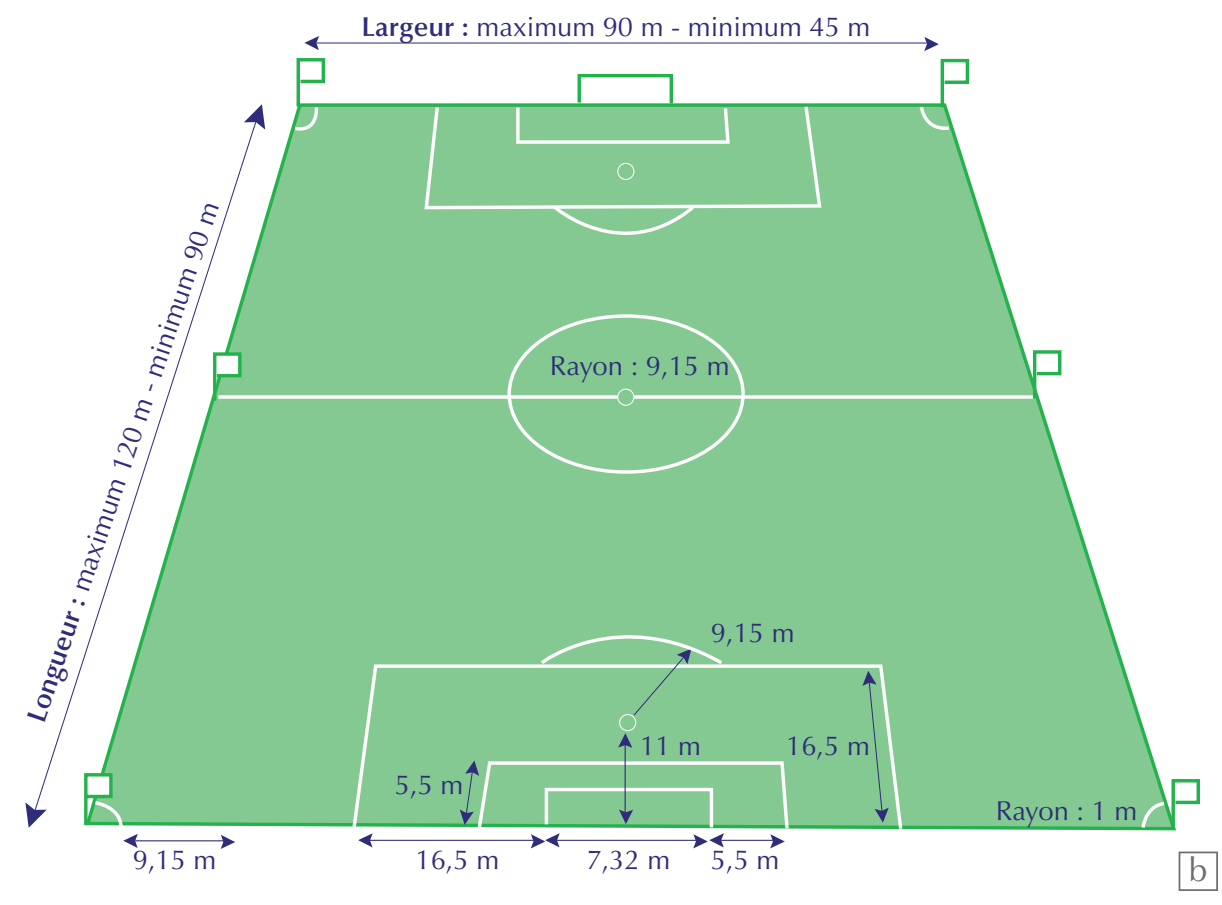




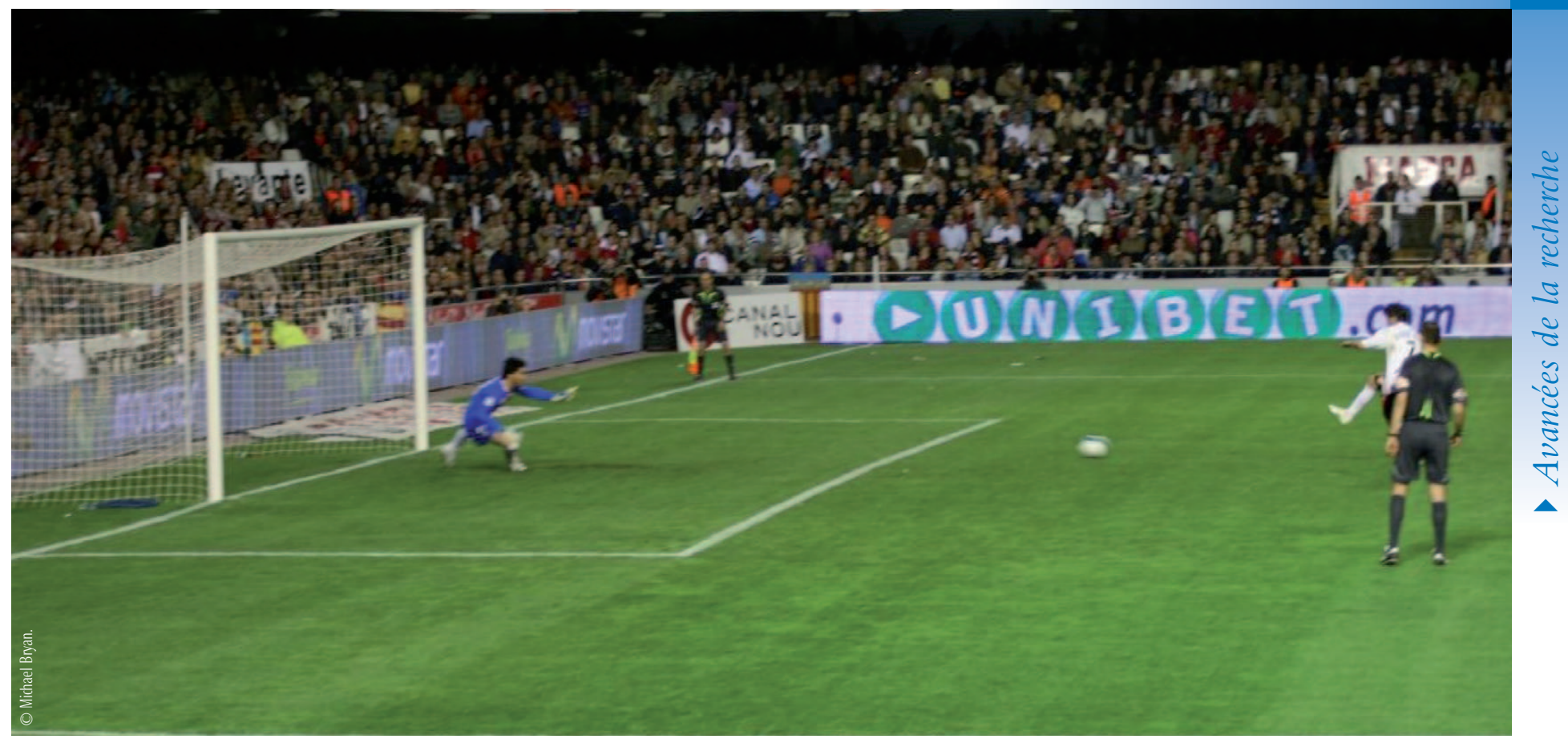

Penalty à 11 mètres.

\section{Diagramme de phase}

Deux forces déterminent la trajectoire du ballon : son poids $\mathrm{F}_{G}$ et la force aérodynamique $\mathrm{F}_{A}$. Dans cette dernière, il est d'usage de distinguer deux composantes, l'une alignée avec la vitesse (traînée $\mathbf{F}_{D}$ ) et l'autre perpendiculaire (portance $\mathrm{F}_{L}$ ). Nous suivons cet usage qui permet, comme nous le verrons, d'isoler l'effet de la rotation du ballon. Ainsi, l'équation générale de la trajectoire devient :

$$
M \mathrm{dU} / \mathrm{d} t=\mathrm{F}_{G}+\mathrm{F}_{D}+\mathrm{F}_{L} \quad \text { (1). }
$$

Dans cette équation, $M$ est la masse du ballon et $\mathrm{U}$ la vitesse de son centre d'inertie. L'origine physique et l'expression des forces $\mathrm{F}_{D}$ et $\mathrm{F}_{L}$ sont détaillées dans l'encadré p. 12 . Nous allons analyser l'équation (1) en identifiant différents régimes caractérisés, chacun, par une trajectoire particulière. Pour cela, nous mesurons l'importance relative des forces avec deux nombres sans dimension : $D_{r}=F_{D} / F_{G}$ et $S_{p}=F_{L} / F_{D}$. Sur la figure 2, nous utilisons ces deux nombres pour définir le diagramme de phase des différentes trajectoires. Lorsque la gravité domine $\left(D_{r}<<1\right)$, on observe des trajectoires paraboliques : c'est le régime des touches ou des lobs (p. 13). À l'inverse $\left(D_{r}>>1\right)$ et en l'absence de rotation $\left(S_{p}<<1\right)$, les trajectoires sont rectilignes ou en zigzag (p. 12). Enfin, lorsque l'aérodynamique domine et que le ballon tourne $\left(D_{r}>>1, S_{p}>>1\right)$, les trajectoires se courbent, comme dans les fameux coup francs de Michel Platini, et se rapprochent de spirales, comme dans celui de Roberto Carlos (pp. 13-14).

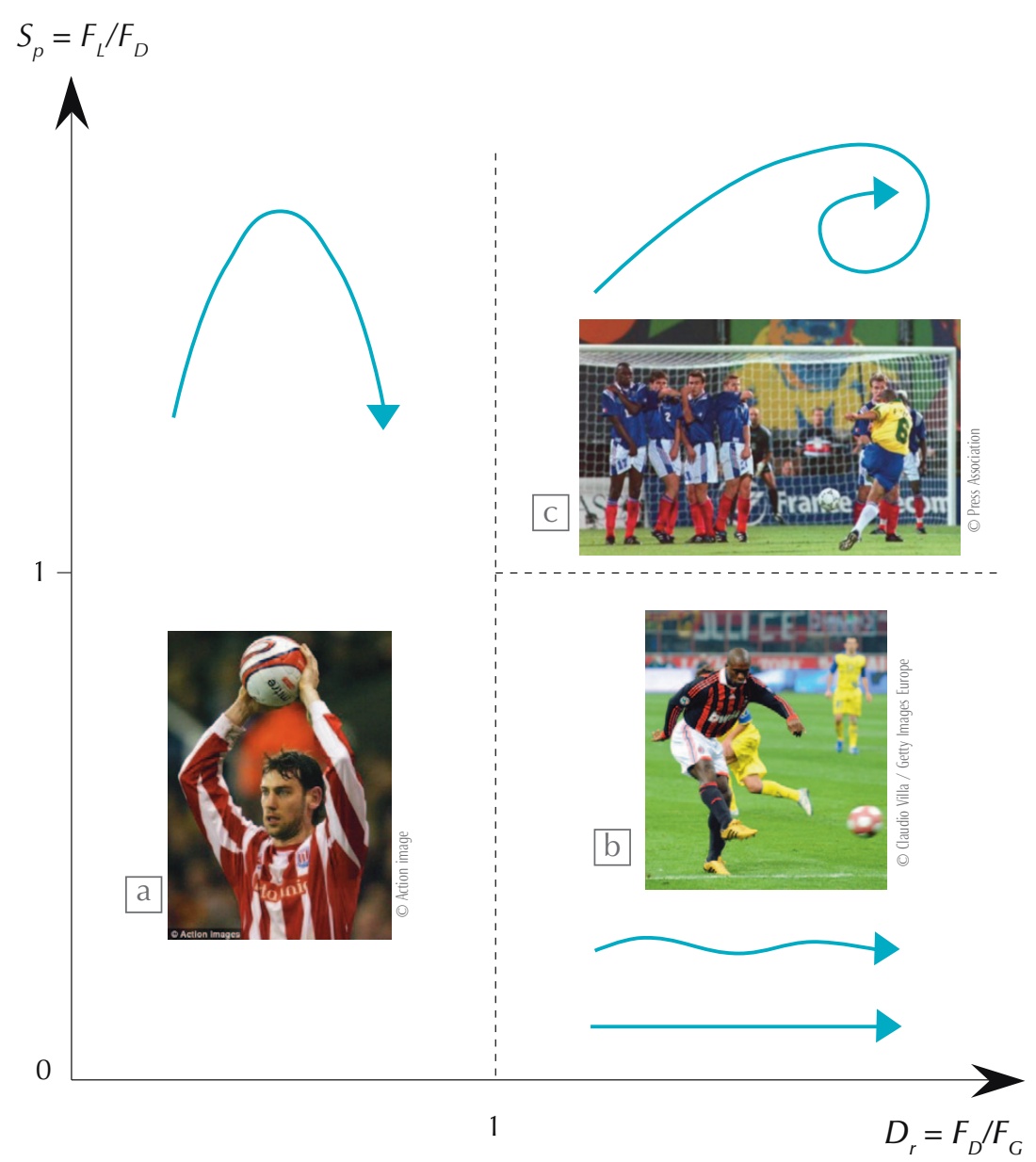

2. Diagramme de phase des différentes trajectoires observées au football. (a) Lorsque la gravité domine $\left(D_{r}<<1\right)$, les trajectoires sont paraboliques. C'est le régime des touches, illustré ici par Rory Delap, joueur irlandais connu pour faire des touches aussi longues que des corners. (b) À plus grande vitesse et en l'absence de rotation $\left(D_{r}>>1, S_{p}<<1\right)$, on observe des trajectoires rectilignes, en zigzag, voire en paraboles tronquées. Ce régime est illustré ici par Clarence Seedorf, footballeur néerlandais connu pour la puissance de ses tirs. (c) Enfin, lorsque l'aérodynamique domine et que le ballon tourne $\left(D_{r}>>1, S_{p}>>1\right)$, on observe des trajectoires courbées, comme dans les fameux coups francs de Platini ou ici de Roberto Carlos. 
$>>$

\section{Lignes droites et zigzags}

On s'intéresse ici aux grosses frappes sans rotation, où $D_{r}>>1$ et $S_{p}<<1$. Dans cette limite, seule la force de trainée $\mathrm{F}_{D} \sim-\rho R^{2} U \mathrm{U}$ influence la trajectoire du ballon. Ici, $\rho \approx 1,2 \mathrm{~kg} / \mathrm{m}^{3}$ est la masse volumique de l'air, tandis que $R=0,11 \mathrm{~m}$ est le rayon du ballon et $U$ sa vitesse. Cette force aérodynamique devient supérieure au poids au-delà de la vitesse critique $U^{\star} \approx 20 \mathrm{~m} / \mathrm{s}$. Le tableau de la page 14 indique des vitesses enregistrées en match lors de grosses frappes.

Ces vitesses sont supérieures à $38 \mathrm{~m} / \mathrm{s}$, ce qui correspond bien au domaine aérodynamique. $\mathrm{F}_{D}$ étant alignée avec la vitesse, on déduit de l'équation (1) que la trajectoire est une droite. De façon plus précise, cette équation s'intègre et l'on montre que la vitesse décroît exponentiellement avec l'abscisse curviligne $s$ suivant la loi $U(s)=U_{0} \exp (-s / \mathcal{f}), \mathcal{E}$ étant la longueur caractéristique de freinage :

$$
E \approx 7\left(\rho_{s} / \rho\right) R
$$

Dans l'équation (2), $\rho_{s}=81 \mathrm{~kg} / \mathrm{m}^{3}$ est la masse volumique du ballon et le facteur 7 est la valeur donnée par le calcul pour nos gammes de vitesses et de tailles. La longueur $\mathcal{E}$ est ainsi de l'ordre de $50 \mathrm{~m}$ pour le football. Tant que $U(s)>U^{\star}$ (typiquement jusqu'à $s \sim \mathcal{E}$ ), les grosses frappes sont donc sensées suivre des lignes droites. On présente sur la figure 3 des trajectoires de ballon observées par Hong et al. [4] pour $U_{0} \approx 28 \mathrm{~m} / \mathrm{s}$ : l'écoulement d'air autour du ballon est visualisé en recouvrant la balle d'une poudre de tétrachlorure de titane.

Dans ces clichés, les petites bouffées révèlent la structure tourbillonnaire complexe à l'aval du ballon et montrent que la trajectoire reste effectivement une quasiligne droite (a), mais qu'elle peut parfois zigzaguer autour de cette droite (b). On observe aussi sur la figure $3 \mathrm{~b}$ que la taille du zigzag est grande comparée à la distance qui sépare deux vortex. Cette séparation d'échelles indique que l'origine du zigzag (aussi observé au volley et au baseball) n'est pas un simple couplage entre le ballon et les tourbillons émis dans son sillage. Son origine physique est encore débattue, mais il semble que la transition turbulente de la couche limite joue un rôle essentiel [3].
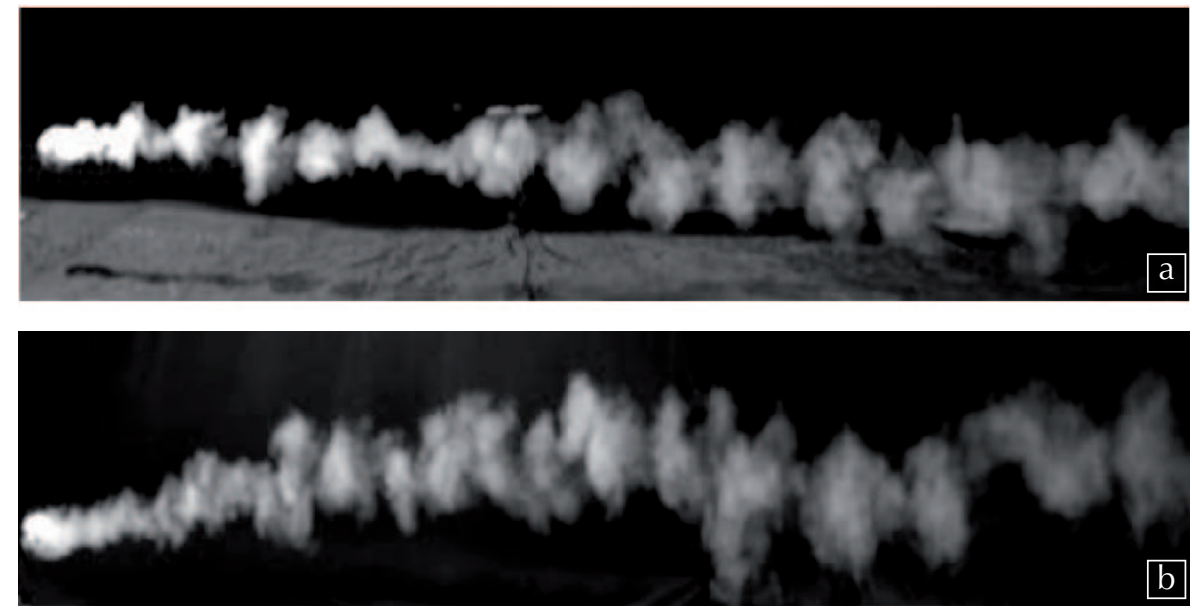

3. Trajectoires de ballons de football observées par Hong et al. pour $U_{0} \approx 28 \mathrm{~m} / \mathrm{s}$ [4].

(a) ligne droite ; (b) zigzag. Sur ces clichés, le ballon va de droite à gauche.

\section{Les forces aérodynamiques}

\section{Origine physique de la traînée}

Lorsque le ballon se déplace à la vitesse $U$ dans de I'air, la forme de l'écoulement n'est pas symétrique entre l'avant et l'arrière. Plus précisément, on voit des tourbillons derrière le ballon, mais pas devant (fig. a). Ainsi, I'air qui est initialement au repos se retrouve en mouvement après le passage du ballon. Il est accéléré, et cette accélération est à l'origine de la force de traînée.

De façon plus précise, on peut estimer cette force en considérant la mise en mouvement de l'air chassé par le ballon, lorsqu'il se déplace d'un diamètre $2 R$. La masse d'air déplacée lors de ce mouvement est contenue dans le cercle en pointillés (fig. a). Cette masse $m$, de l'ordre de $\rho R^{3}$ (où $\rho$ est la masse volumique de l'air), passe $d^{\prime}$ une vitesse nulle à $U\left(\Delta U_{\|} \sim U\right)$ sur un temps $\Delta t \sim R / U$. La variation de quantité de mouvement par unité de temps, $m \Delta U_{\|} / \Delta t$, conduit à l'expression de la force de traînée $F_{D} \sim \rho R^{2} U^{2}$.

\section{Origine physique de la portance}

Lorsque le ballon tourne avec la vitesse angulaire $\omega$, les tourbillons qui sont émis n'ont plus la même intensité : celui qui est entraîné par la rotation tourne plus vite, tandis que I'autre est ralenti (fig. b). Cet effet implique que l'air est plus accéléré d'un côté que de I'autre, ce qui fait apparaître une force perpendiculaire à l'écoulement, dite " force de Magnus ». Pour trouver son expression, on raisonne comme précédemment, en considérant cette fois la variation de vitesse dans la direction perpendiculaire à l'écoulement : $\Delta U_{\perp} \sim R \omega$, d'où $F_{L} \sim m \Delta U_{\perp} / \Delta t \sim \rho R^{3} \omega U$.
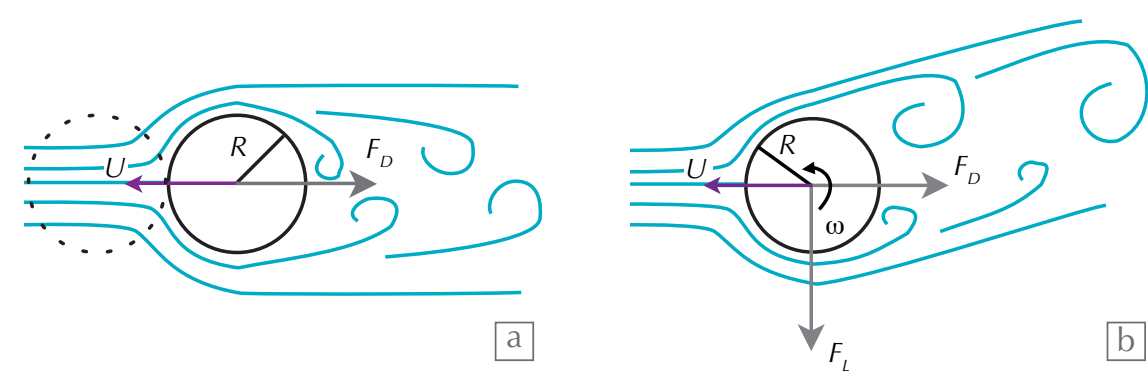

Écoulement de l'air autour d'un ballon se déplaçant à une vitesse $U$, (a) sans rotation, (b) avec rotation. 


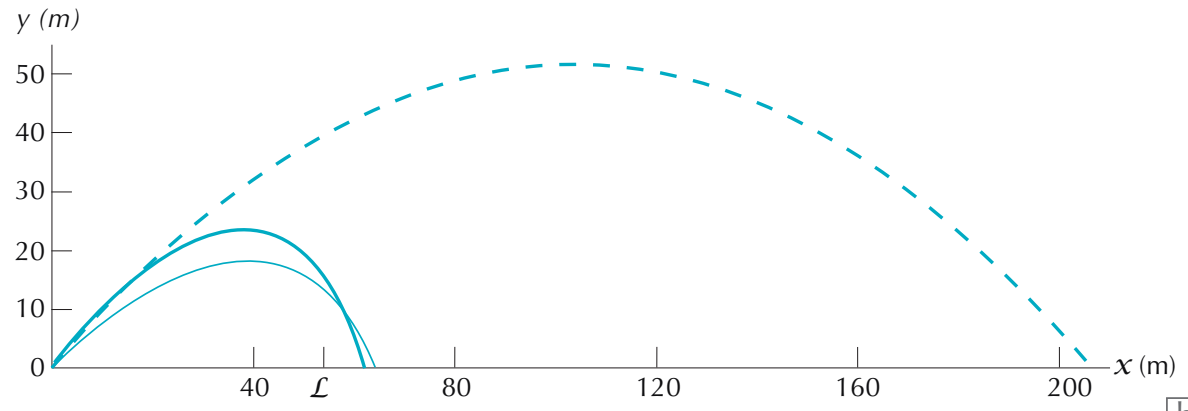

4. Dégagements. (a) Les dégagements des gardiens de but (ici, Steve Mandanda) dépassent en général de peu le milieu de terrain, et sont caractérisés par une courbe en cloche fortement asymétrique par rapport à leur apogée. (b) Trajectoires du ballon avec gravité et freinage aérodynamique (trait plein) et avec la gravité seule (pointillés). La vitesse initiale est $U_{0}=45 \mathrm{~m} / \mathrm{s}$, l'angle initial est $45^{\circ}$ pour le trait épais et $37^{\circ}$ pour la trajectoire la plus longue (trait mince). Pour ces deux cas, $£=54 \mathrm{~m}$.

(Source de la photo : http://fansdemarseille.centerblog.net/6-steve-mandanda ).

\section{Paraboles et paraboles tronquées}

Lorsque la distance parcourue par le ballon est supérieure à la distance de freinage $\mathcal{E}$, la vitesse du ballon diminue et la gravité ne peut plus être négligée dès lors que $U$ devient inférieure à $U^{\star}$. Cette situation se rencontre lors des dégagements des gardiens de but (fig. 4a). Dans cette limite, nous avons intégré numériquement l'équation (1), en prenant en compte $\mathrm{F}_{D}$ et $\mathrm{F}_{G}=M \mathrm{~g}$. Les trajectoires obtenues pour deux angles initiaux différents avec $\mathcal{E}=54 \mathrm{~m}$ et $U_{0}=45 \mathrm{~m} / \mathrm{s}$ sont présentées sur la figure $4 \mathrm{~b}$. On observe sur cette figure un écart important avec la parabole galiléenne : quand on atteint la longueur $f$, l'inertie du ballon ne compte plus, si bien qu'il retombe presque verticalement. On voit aussi que l'angle qui maximise la distance n'est plus $45^{\circ}$ mais $37^{\circ}$. Cet angle optimal varie avec $\mathcal{E}$, et $U_{0}$.

\section{La spirale}

Nous retenons des deux sections précédentes que, dans la limite des grandes vitesses $\left(U_{0}>>U^{\star}\right)$, la gravité peut être négligée, et la trajectoire est rectiligne sur des distances petites devant $\mathcal{E}$. Ceci est vrai en l'absence de rotation. Avec rotation, le ballon subit aussi la force de Magnus $\mathrm{F}_{L} \sim \rho R^{3} \omega_{0} \wedge \mathrm{U}$, où $\omega_{0}$ est le vecteur rotation. Cette force, perpendiculaire à la vitesse, dévie le ballon de la ligne droite. Plus précisément, la courbure locale de la trajectoire $C(s)$ évolue suivant l'équation $M U^{2} C(\mathrm{~s})=F_{L}$. En utilisant l'expression de $F_{L}$ et en prenant en compte la décroissance exponentielle de la vitesse, cette équation indique que la courbure de la trajectoire augmente à partir de l'impact suivant la loi :

$C(s) \sim\left(\rho / \rho_{s}\right)\left(\omega_{0} / U_{0}\right) \exp (s / \mathcal{L}) \quad(3)$.

Cette trajectoire a été étudiée en détail par Dupeux et al. [5], et nous ne discutons ici que ses implications pour le football. L'équation (3) montre que la trajectoire est une spirale dont la courbure initiale $C_{0} \sim \rho \omega_{0} / \rho_{\mathrm{s}} U_{0}$ augmente exponentiellement sur la longueur de freinage $\mathcal{E}$.

Nous discutons sur la figure 5 la possibilité d'observer cette spirale dans le cas de trois coups de pied arrêtés. À l'échelle du penalty (fig. 5a), la distance de $11 \mathrm{~m}$ est petite comparée à la taille de la spirale, et seul le début rectiligne de la trajectoire est observable. Pour un coup franc aux $18 \mathrm{~m}$ (fig. 5b), le cœur de la spirale n'est pas

5. Trois types de coups de pied arrêtés. (a) Penalty à $11 \mathrm{~m}$; (b) coup franc « aux 18 mètres » ; (c) coup franc lointain. 


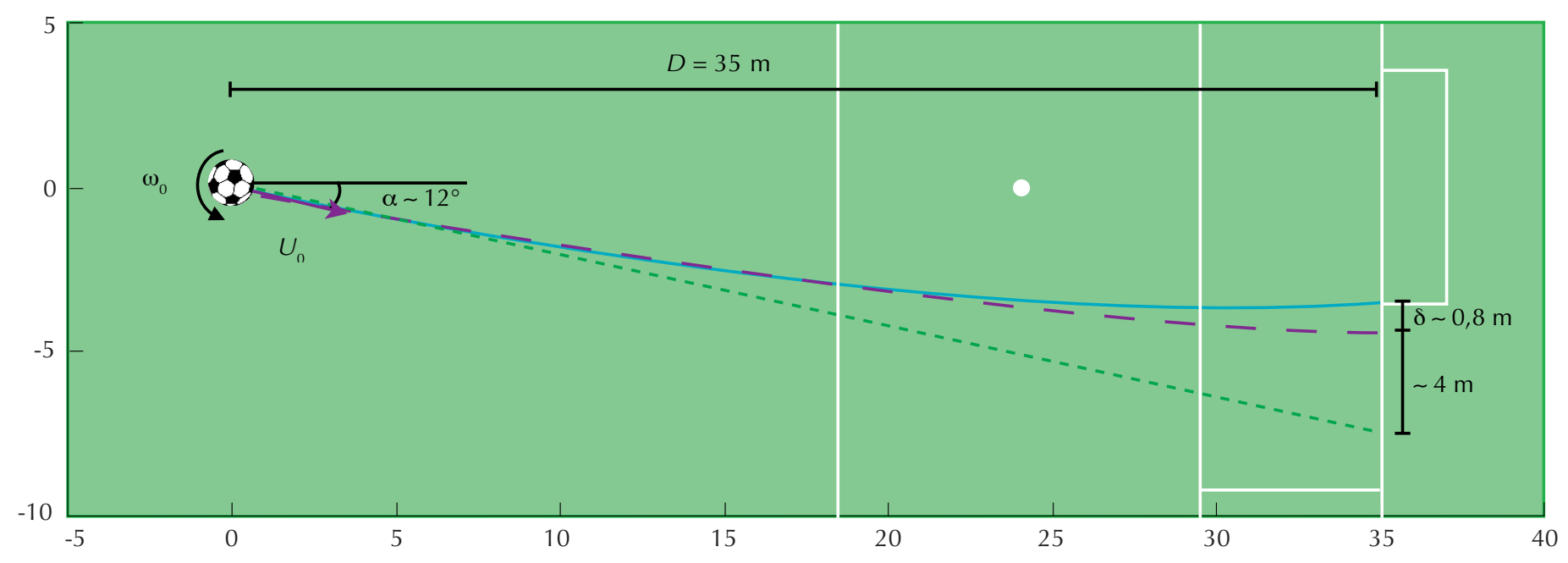

6. Coup franc de Roberto Carlos, tiré contre la France en 1997. On détaille ici le coup franc présenté sur la figure 5c. La trajectoire bleue est calculée à partir de l'équation (3), avec $U_{0}=38 \mathrm{~m} / \mathrm{s}$ et $\omega_{0}=88 \mathrm{rad} / \mathrm{s}$. La trajectoire violette est le cercle de courbure $C_{0}$, et le trait pointillé vert indique la trajectoire rectiligne.

\section{\〉}

accessible, mais la trajectoire est courbée et on peut l'estimer par un cercle de courbure $C_{0}$. Pour un coup franc lointain (fig. 5c), la trajectoire courbée dévie du cercle et l'augmentation de courbure devient observable. Selon nous, cette augmentation est à l'origine de la trajectoire surprenante observée lors du coup franc de Roberto Carlos tiré contre la France en 1997 [6]. Ce coup franc est schématisé sur la figure 6 : il est tiré des 35 mètres à une vitesse $U_{0}=38 \mathrm{~m} / \mathrm{s}$ et un angle initial $\alpha$ de l'ordre de $12^{\circ}$. Du fait de la rotation $\left(\omega_{0} \approx 88 \mathrm{rad} / \mathrm{s} \approx 15\right.$ tours par seconde $)$, la trajectoire du ballon s'incurve et finit par entrer dans le but sans que le gardien bouge (courbe bleue). Sans rotation, le ballon sortirait de 4 mètres (pointillés). Avec une trajectoire circulaire, le ballon manque la cage de 80 centimètres (tirets violets). Du fait de la spirale, le ballon s'écarte de la trajectoire circulaire dans les derniers mètres qui le séparent du poteau et finit par entrer, à la surprise générale.

Dans le cas du coup franc de Roberto Carlos, l'axe de rotation est vertical et le ballon dévie horizontalement vers la gauche, ce qui lui permet de contourner le mur. Un autre type de coup franc est celui où le tireur décide de faire passer le ballon par-dessus le mur et de le faire plonger ensuite. Pour cela, il choisit un axe de rotation horizontal et fait tourner le ballon de telle sorte que son sommet aille vers l'avant (tel un lift au ping-pong). Ce type de coup franc est rare, car il demande un petit pied, suffisamment petit pour passer sous le ballon et le brosser. Un des derniers grands maîtres capables de réaliser ce geste est Juninho Pernambucano [7].

\section{Conclusion}

Gravité et aérodynamique sont responsables de la diversité des trajectoires du ballon de football. À faible vitesse (touche, lob), la gravité domine et seule la parabole galiléenne est observée. À forte vitesse (penalty, coup franc, tir tendu), l'aérodynamique devient prépondérante et la trajectoire se met à dépendre de la rotation $\mathrm{du}$ ballon. Sans rotation, celui-ci va tout droit ou zigzague, suivant la nature laminaire ou turbulente de sa couche limite. Cette trajectoire quasi rectiligne perdure, jusqu'à ce que le ballon soit suffisamment ralenti pour que la gravité le fasse tomber, donnant lieu à des paraboles tronquées. Le dernier régime est celui des ballons en rotation qui conduit à des cercles, voire à des morceaux de spirale lorsque la distance parcourue est suffisament grande. I

Vitesses de ballon enregistrées lors de grosses frappes.

\begin{tabular}{|l|l|l|}
\hline Joueur & $\mathbf{U}_{\mathbf{0}}(\mathbf{m} / \mathbf{s})$ & Match \\
\hline David Hirst & 51 & Sheffield / Arsenal (16/09/96) \\
\hline David Beckham & 44 & Manchester Utd / Chelsea (22/02/97) \\
\hline David Trézéguet & 43 & Monaco / Manchester Utd (19/03/98) \\
\hline Richie Humphreys & 42,8 & Sheffield / Aston Villa (17/08/96) \\
\hline Matt Le Tissier & 39 & Southampton / Newcastle (18/01/97) \\
\hline Alan Shearer & 38,3 & Newcastle / Leicester (02/02/97) \\
\hline Roberto Carlos & 38,1 & Brésil / France (03/06/97) \\
\hline
\end{tabular}

\section{Références}

1• W.D. Hill, M. Blake et J.E. Clark, "Ball court design dates back 3,400 years", Nature 392 (1998) 878.

2• C. Reep et B. Benjamin, "Skill and Chance in Association Football", Journal of the Royal Statistical Society, Series A (General) 131 (1968) 581-585.

3• A. Armenti (ed.), The physics of sports, AlP-Press, Springer-Verlag (1992).

4• S. Hong, C. Chung, M. Nakayama et T. Asai, "Unsteady Aerodynamic Force on a Knuckleball in Soccer", Procedia Engineering 2 (2010) 2455-2460.

5• G. Dupeux, A. Le Goff, D. Quéré et C. Clanet, "The spinning ball spiral", New Journal of Physics 12 (2010) 093004.

6• www.youtube.com/watch?v=crSkWalqx-Y

7• www.youtube.com/watch?v=DTeFKTnEyzY 